\title{
Three-dimensional PIC/MC simulations of the sustain discharge pulse in an ACPDP
}

\author{
V. N. Khudik \\ V. P. Nagorny \\ A. Shvydky
}

\begin{abstract}
The strong discharge pulse between coplanar electrodes in an ACPDP cell is investigated using fully kinetic 3-D simulations. Key phases in the discharge development are identified and thoroughly illustrated with an extensive set of plots. The main effort is focused on the study of the anode charging wave and striations formed above the dielectric surface in the anode area. To elucidate these physical phenomena, we perform a number of specially designed numerical experiments.
\end{abstract}

Keywords - ACPDP, PIC/MC simulation, PDP discharge, striations, cathode fall.

\section{Introduction}

Numerical simulations based on the hydrodynamic approximation proved to be a very useful tool for studying discharges in a plasma-display-panel (PDP) cell, and currently are widely used. ${ }^{1-4}$ They have provided very valuable information about the time-spatial evolution of the discharge (such as distribution of the electric field, electrons, ions, and excited species in the cell), and allowed us to investigate the dependence of different discharge parameters on the dielectric properties of the walls, electrode structure, gas mixture, etc.; they also gave clues for the development of analytical theories. ${ }^{5,6}$ The advantage of a numerical approach is that it allows one to "look" inside the discharge and find relationships between discharge characteristics, which are hard or impossible to extract from experimental data. Moreover, one can even deliberately change the value of any physical parameter in the simulation in order to uncover some "hidden" relationships. All of this helped to form basic concepts and to understand many features of the PDP discharge, and significantly improve its parameters.

One should remember, though, that hydrodynamic approximation requires too many assumptions (which do not have a solid theoretical foundation) about the electron distribution function under the conditions of the PDP discharge. Indeed, while the electric field in the PDP cell varies very sharply in space and very fast in time, transport coefficients and rates of excitation and ionization processes used in fluid models were obtained for a uniform and stationary electric field (through independent kinetic zero-dimensional considerations!). So, at most, one should consider fluid simulations, although extremely useful, as giving a qualitative picture of the discharge, rather than quantitative. (Note that recent modifications of fluid $\operatorname{codes}^{3,4,7}$ which include a separate equation for the electron energy are capable of capturing some non-local discharge features; however, whether or not they produce more accurate quantitative results is still an open question.)
The strength of the kinetic approach is that it uses only fundamental data, such as cross-sections of different interactions between all kinds of particles, and probability distribution functions of various particle-wall interactions. The distribution of relevant particles is calculated self-consistently during discharge development at every point in time and space without any apriori assumptions. The obvious difficulty of using kinetic codes is the significantly longer computational times compared to those of fluid ones. That is why kinetic simulations of a PDP discharge were rare, and limited to, at most, two-dimensional geometries. ${ }^{8}$ However, 2-D kinetic codes do not take into account certain geometrical features of a real PDP cell (such as barrier ribs, width of the address electrode, etc.) and cannot correctly reproduce a spatial structure of the discharge along the third dimension (particularly, the shape of discharge striations and the ionizing cathode wave observed in experiments). This problem was finally solved in our 3-D Monte-Carlo/PIC kinetic simulations described in this paper.

\section{Particle-In-cell/Monte-Carlo algorithm}

We have chosen the PIC/MC method rather than the one based on the Boltzmann equation for the electron distribution function because for the PDP conditions PIC/MC is much faster and is the only method that correctly approaches statistical fluctuations - important, for example, for analysis of jitter ${ }^{9,10}$ or weak discharges with a small number of charged particles.

The speed advantage of the PIC/MC method comes from the simple fact that the electron distribution function in the Boltzmann equation is defined in a six-dimensional space, and its discretization with any reasonable accuracy would require manipulation with huge arrays (with, at least, $10^{9}-10^{10}$ elements), even if there are only a few electrons in the PDP cell. On the other hand, even during a strong sustain discharge pulse, the number of charged particles transferred in a typical PDP cell is only of the order of $10^{8}$.

Received 11/02/04; accepted 11/29/04.

V. N. Khudik and V. P. Nagorny are with Plasma Dynamics Corp., 1004 Waterville-Monclova Rd., Waterville, OH 43566; telephone 419/297-4724, fax 419/878-3820, e-mail: vnagorny@plasmadynamics.com

A. Shvydky is with the University of Toledo, Toledo, OH, U.S.A.

(C) Copyright 2005 Society for Information Display 1071-0922/05/1302-0147\$1.00 


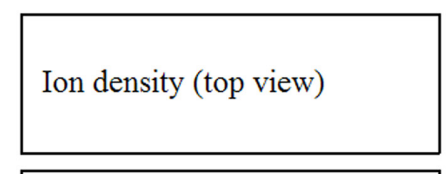

Charge deposition rate on
the dielectric surface above
sustain electrodes (top view)

$\mathrm{t} 1=28.6 \mathrm{~ns}$
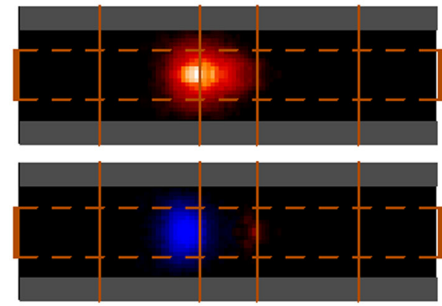

$\mathrm{t} 2=83.6 \mathrm{~ns}$
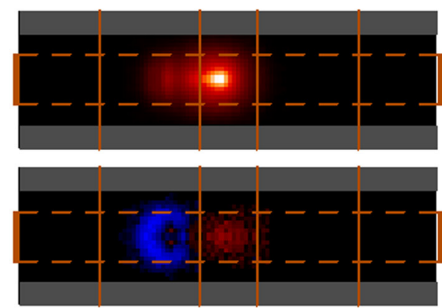

$\mathrm{t} 3=97.6 \mathrm{~ns}$
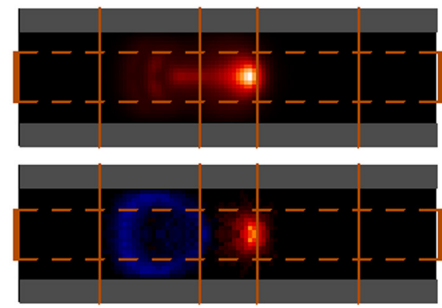

$\mathrm{t} 4=113.5 \mathrm{~ns}$
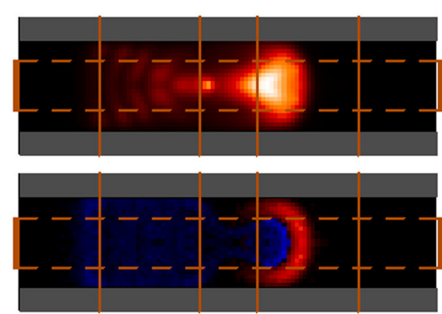

$\mathrm{t} 5=144.7 \mathrm{~ns}$
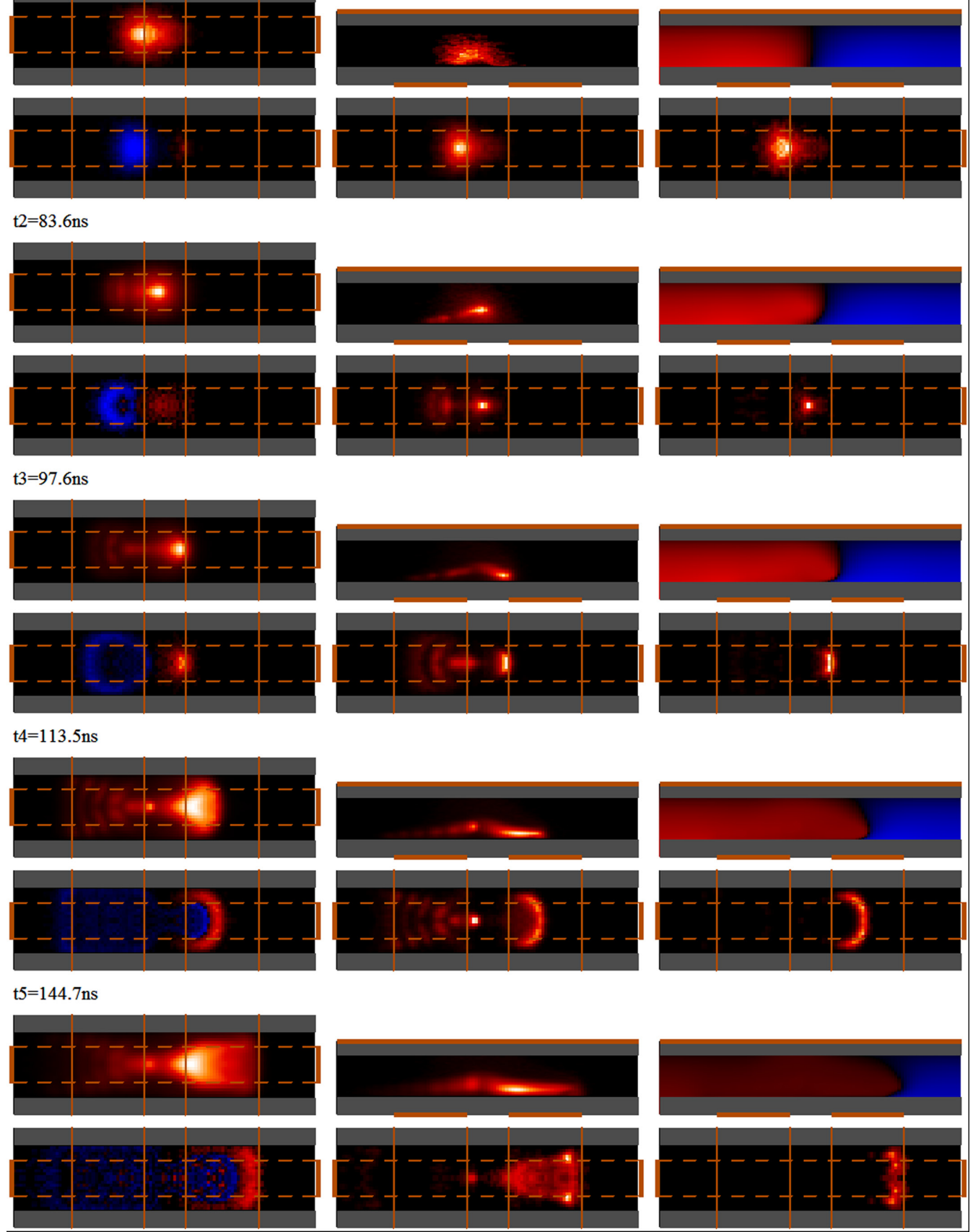

FIGURE 1 - Spatial distribution of discharge characteristics at different moments of time. Each time-frame includes six windows whose contents are explained in the top sketch. Red color corresponds to positive values, blue color to negative ones. 
Without sacrificing accuracy, one can use relatively small positive/negative macro-particles (each of them representing up to a few tens of real ions/electrons) and limit the number of values recalculated at each time step to about $10^{6}$. So, the speed advantage can be clearly on the order of $10^{3}-10^{5}$.

In our simulations, each macro-particle corresponds to 50 electrons/ions and the total number of particles in the PDP cell has not exceeded two million. In order to accurately reproduce the electron trajectories, electrons are advanced with a time step less than $10^{-5}$ nsec. To simulate the electron collisions, we have used a null-collision technique with a set of cross-sections similar to that of Ref. 1 . The standard chemical model of the Xe/Ne-mixture discharge has been used to track the evolution of the excited species.

Poisson's equation is solved on a 3-D rectangular mesh with a cell size of about several microns (by one of the relaxation methods). To prevent plasma from the artificial numerical heating, the electric potential is recalculated with a time step $\Delta t=\left(\omega_{p e} e^{-1}+\tau_{m}\right) / k$, where $\omega_{p e}$ is the electron plasma frequency, $\tau_{m}$ is the electron Maxwellian time, and $K$ is some numerical coefficient (see Appendix).

\section{Dynamics of strong discharge pulse in a PDP cell}

In this section we describe general features of a strong discharge pulse in an ACPDP cell which weakly depends on the specifics of the discharge parameters. We considered a standard coplanar PDP cell, filled with a neon-xenon (93\% - 7\%) mixture at a pressure of 500 torr. The cell length, width, and height are 650, 220 (including barrier ribs), and $160 \mu \mathrm{m}$ (including dielectric layers above sustain and address electrodes with dielectric permeability equal to 11 ), respectively. The distance between sustain electrodes is 90 $\mu \mathrm{m}$, the sustain electrode width is $155 \mu \mathrm{m}$, and the voltage applied to the sustain electrodes is $\pm 220 \mathrm{~V}$. The secondary electron emission coefficients for $\mathrm{Ne}$ and $\mathrm{Xe}$ ions are 0.5 and 0.01 , respectively.

Since the distance between sustain electrodes is small, the barrier discharge in an ACPDP cell develops mainly near the dielectric surface above the anode and the cathode. It goes through the following stages (illustrated in Fig. 1).

Phase 1. At first, the positive charge is accumulated in the gap volume above the inner edge of the anode electrode and at the same time the negative charge is deposited on the dielectric surface. The charge created on this stage is still small and the distribution of the electric potential is almost the same as in empty gap (see Fig. 1, the moment $t$ $\left.=t_{1}\right)$.

Phase 2. Then, when the density of the positive charge reaches a certain critical level, ions starts to screen the electric field and detain the electrons in the gap volume. As a result, a plasma region forms above the inner edge of the anode and then gradually protrudes toward the cathode (see Fig. 1, the moment $t=t_{2}$ ). There is a substantial positive charge on the tip of this plasma region (much like the charge on the tip of the conductor placed in an external electric field). At this stage, ongoing deposition of the negative charge on the anode dielectric surface is accompanied by the formation of first striations (see "ion density" and "Xe-excitation rate" windows in Fig. 1, the moment $t=t_{2}$ ), and the deposition of the positive charge on the cathode dielectric surface is still insignificant.

Phase 3. When the plasma region approaches the dielectric surface above the inner edge of the cathode (see Fig. 1, the moment $t=t_{3}$ ), the cathode fall (CF) is formed, and the current through the low-resistive plasma channel sharply increases. Since the potential drop between the plasma region and the uncharged areas of the dielectric surface is substantially higher than the breakdown voltage, the $\mathrm{CF}$ expands along the cathode in the form of an ionizing wave, and the positive charge is deposited on newer and newer areas of the dielectric surface. (The ionizing wave is readily identified by the red arc in the "charge deposition rate," "Xe-excitation rate," and "Ne-excitation rate" windows in Fig. 1, the moment $t=t_{4}$.) Directly behind this wave, the deposition of the positive charge sharply decreases and becomes overshadowed by the deposition of the negative charge. In this area, hot electrons diffuse against (or across) the electric field from the plasma toward the surface.

In our simulations, the speed of the ionizing wave was several times less than the characteristic drift velocity of the ions in the CF region. Note that this result is quite natural. Indeed, under conditions when seed electrons (which initiate electron avalanches) in the CF are created only by the secondary electron emission due to ion impacts on the surface (i.e., when other processes such as photoemission, photoionization, etc., can be neglected), the velocity of the ionizing wave must be less than the maximum ion drift velocity in order to sustain a source of seed electrons ahead of the CF.

Phase 4. When the positive charge covers most of the dielectric surface above the cathode (see Fig. 1, the moment $t=t_{5}$ ), the discharge extinguishes. In the afterglow, the number of ions and electrons in the PDP cell gradually decreases through the dissociative recombination of electrons and $\mathrm{Xe}_{2}{ }^{+}$molecules. Charged particles are also pulled out of the plasma toward the dielectric surfaces by the residual (and ambipolar) electric fields.

Note that the atoms of neon are excited almost exclusively in the area of the cathode (where the electric field is relatively strong), whereas the excitation of xenon atoms takes place in both the anode and cathode areas (see Fig. 1, $\mathrm{Ne}$ - and Xe-excitation rates). This difference is also observed in experimental data. ${ }^{11,12}$

The deposition of the negative charge on the dielectric surface in the anode area initially progresses in a wave-like manner, with a quite pronounced wavefront of a distorted circular shape (within which the plasma striations are formed). But with time, this deposition becomes much 

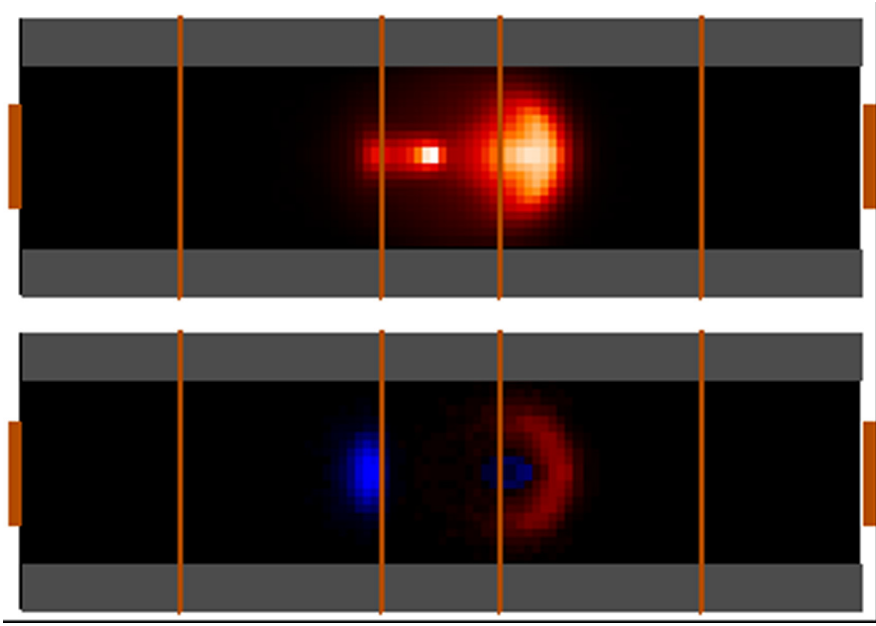

FIGURE 2 - Distribution of the ion density (top view) and charge deposition rate in the surface of the discharge when the dielectric above the anode is replaced by a metal (red color corresponds to positive values, blue color to negative ones).

more uniform (compare the blue areas above the anode in the "charge deposition rate" window in Fig. 1 at time moments $t_{4}$ and $t_{5}$ with those at time moments $t_{2}$ and $\left.t_{3}\right)$. In other words, with time the whole anode area gets directly involved in closing the electric circuit between the anode and the cathode.

In the next section, we describe results of specially designed numerical experiments which help get a better understanding of physical phenomena occurring in the anode area of the PDP cell (see, also, a worthwhile treatment $^{8}$ ).

\section{$4 \quad$ Numerical experiments}

In the first experiment, we replaced the dielectric directly above the anode by a material with high electric conductivity. In this case, the same ionizing wave propagates above the cathode, but deposition of the negative charge occurs within a small area above the inner edge of the anode electrode (see, Fig. 2). Now, there is no component of the electric field parallel to the surface, and electrons cannot be pulled to the new surface areas; so that ionizations (and excitations) occur above the anode almost in the same place. As a result, above the metal there are no near-surface striations at all; they are formed only above the dielectric surface, along which a non-zero component of the electric field exists.

In the second numerical experiment, we artificially made ions immobile in the left-hand side of the PDP cell (and do not introduce any changes in the right-hand side, see Fig. 3). As one can see from Fig. 3, the formation of striations is not a result of ion movements (contrary to what was suggested in Ref. 13): "plasma stripes" near the dielectric surface become even more distinct when the ion velocity is set to zero! The motion of the ions in the electric field leads to smearing out of the spatial modulation of the

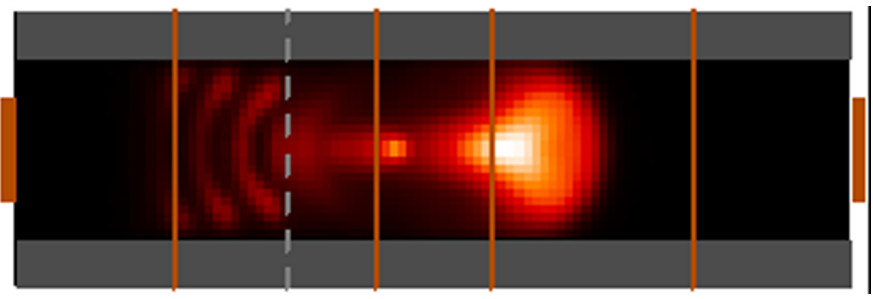

FIGURE 3 - Distribution of the ion density (top view) when ion velocity is artificially set to zero in the left-hand part of the PDP cell (on the left from the dashed gray line).

charged particle densities (as can be noticed in the "ion density" windows in Fig. 1, the moment $t=t_{5}$ ). Overall, one can say that positively charged ions play a passive role in the phenomenon considered.
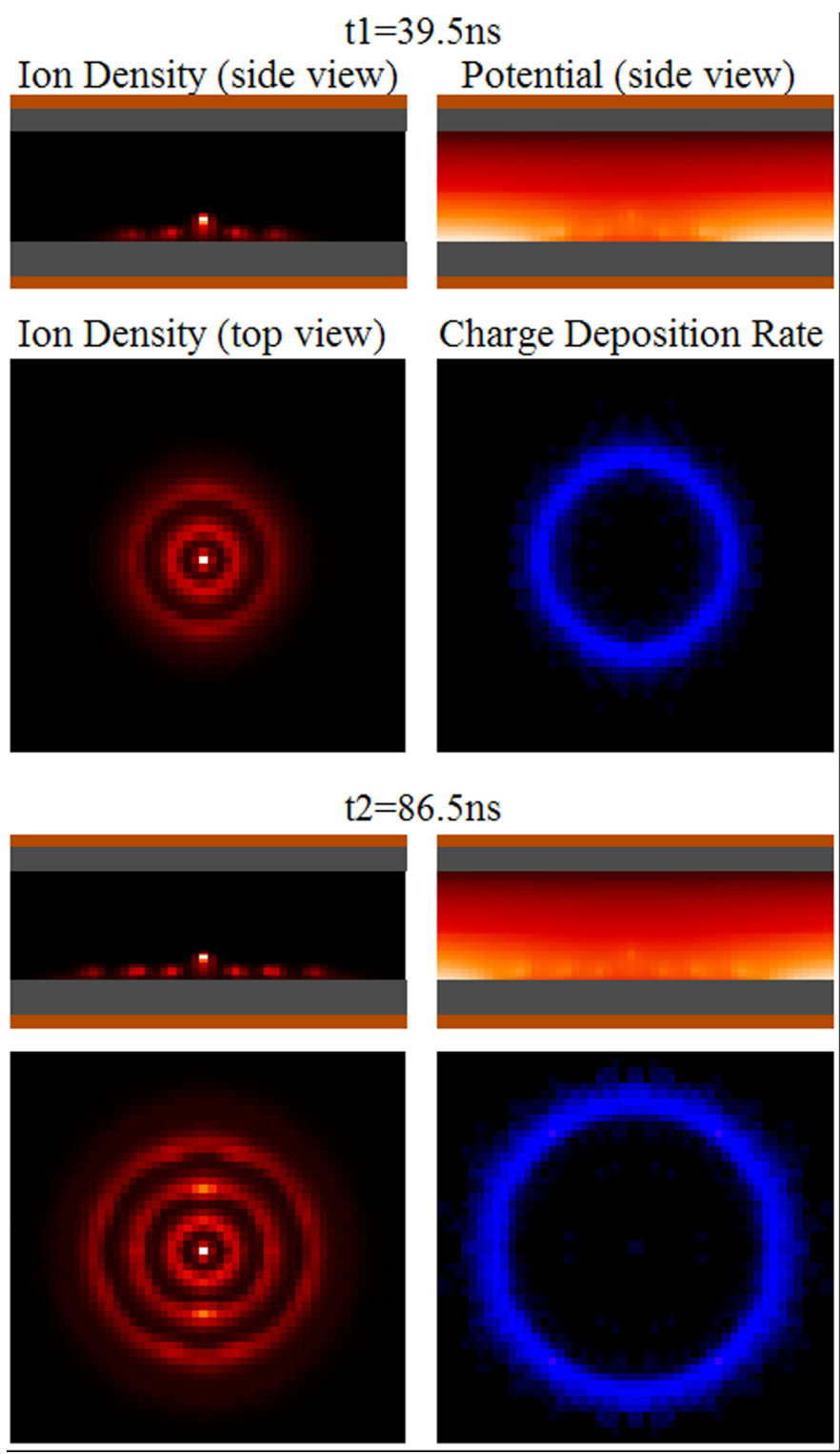

FIGURE 4 - Spatial distribution of discharge characteristics at different moments of time in the system composed of two opposing electrodes (red color corresponds to positive values, blue color to negative ones). The position of the injection spot of electrons is identified by the brightest point in each "ion density" window. 

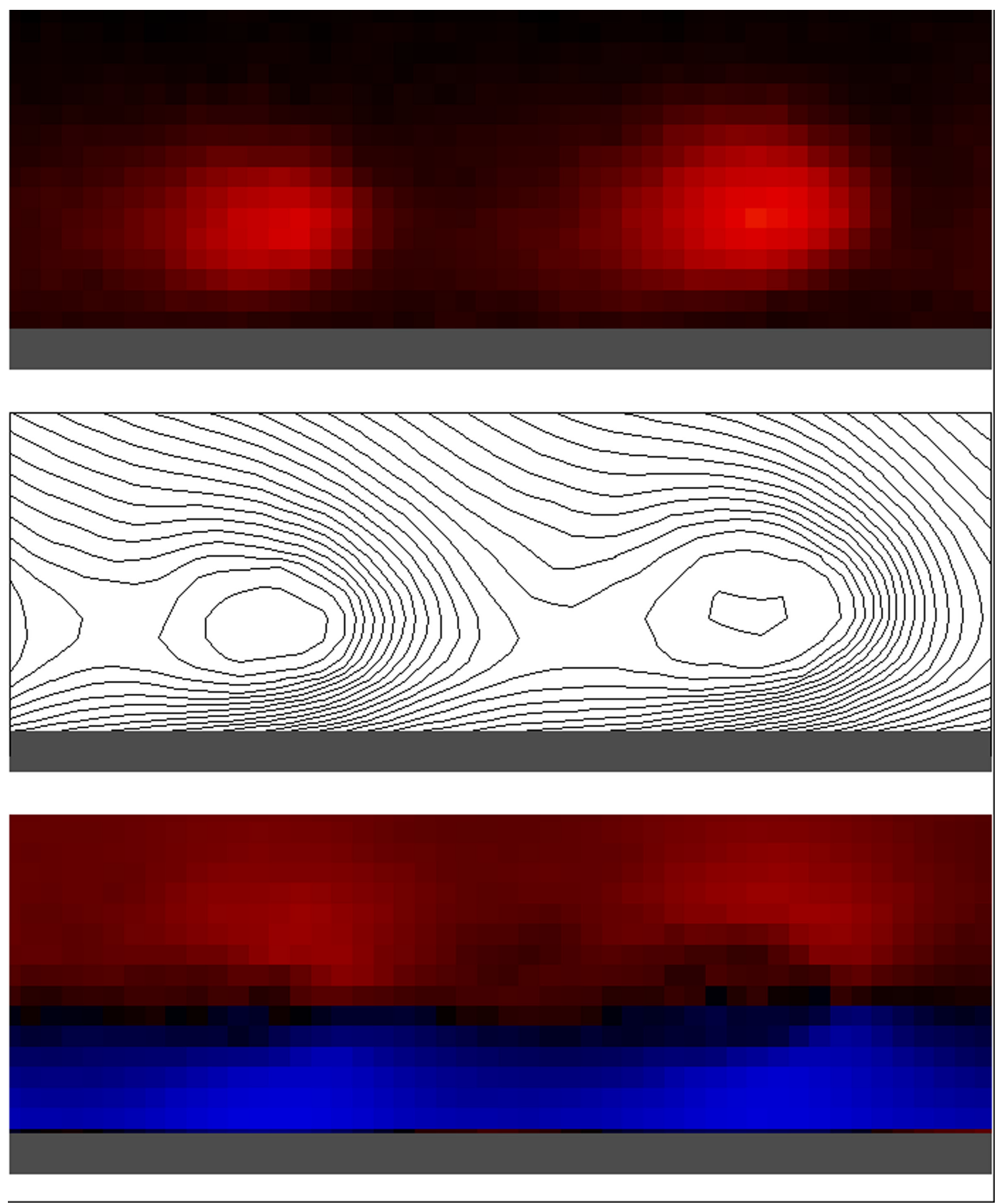

FIGURE 5 - Side view of the ion density (top), electric potential (middle), and vertical component of the electric field (bottom) within the area that includes two consecutive striations located to the left of the injection spot of electrons (red color corresponds to positive values, blue color to negative ones; the gray strip on the bottom of each plot denotes part of the dielectric layer above the anode electrode). The voltage between the equi-potential contours in the middle plot is equal to $1 \mathrm{~V}$.

In the third experiment, we make a number of radical geometrical simplifications (see Fig. 4). In order to separate the physical phenomena near the anode from those near the cathode, we consider the formation of striations in the system composed only of the unbounded upper address electrode and the unbounded lower anode electrode covered by dielectric layers (with thickness $d$ and dielectric constant $\varepsilon$ ). The anode is under positive potential $V_{0}$ so that, initially, there is only a vertical uniform electric field in the gap, $E_{0}$
$=V_{0} / L_{0}$ (where $L_{0}$ is the vertical distance between address and anode electrodes). We also exclude the ion movements, setting the velocity of all ions to zero. In order to imitate the electron source from the plasma channel in standard geometry, we inject electrons within a small area right above the surface of the anode dielectric. As one can see from Fig. 4, the plasma striations and the front of the charging wave have a ring-like shape (as should be expected in the geometry without a preferred horizontal direction). 
At the beginning of the injection, the electrons drift down in the vertical electric field, and the negative charge is deposited on the lower dielectric surface right under the injection spot. With time, this negative charge reverses the vertical component of the electric field near the surface and creates its horizontal component (and a potential difference between already charged and still uncharged surface areas, as clearly seen in the "potential" windows in Fig. 4). The redirected electric field pulls electrons to new areas of a bigger radius. Note that ions created (by the ionization processes) in the gap above the dielectric surface strongly influence the processes of transporting the electrons to uncharged areas of the surface.

Our simulations show that the result displayed in Fig. 4 is not sensitive to the time dependence of the injection current $I(t)$ (providing this current is not too strong). The key parameter appears to be the charge $Q$ of the time integrated current: the radius of the charging wavefront (and number of striations) depends on the amount of charge $Q$ already deposited on the surface rather than directly on time $t$ (in a sense, the time is excluded from our problem!). The instant velocity of the front, therefore, is controlled by the current $I(t)$.

When the distance between upper and lower electrodes is great and the thickness of the dielectric layer above the lower electrode is small, in our super simplified geometry there are only two external parameters which influence the charge deposition process: the initial electric field $E_{0}$ and the effective dielectric thickness $d / \varepsilon$. We have found that the smaller the latter parameter, the slower the deposition process (i.e., when we took two times smaller $d / \varepsilon$, we had to inject approximately two times more electrons in order to get the same striation picture).

In the forth experiment (performed in the same geometry as the previous one), we focus our attention on the fine structure of striations. Although our simulations do not allow us to come to unambiguous quantitative conclusions, we think that it is useful to show a qualitative behavior of the potential lines and the vertical component of the electric field between two consecutive striations (see Fig. 5).

Although we tried several different energy distributions of the injected electrons (and got the similar results), it is not clear now how this distribution influences the formation of the striations. For example, is there any distribution of the injected electrons at which there are no striations at all? There is also no clear understanding of the role of the electrons trapped by the positive charge of the ion striations.

\section{Conclusions}

In a strong discharge in an ACPDP cell with a small distance between sustain electrodes, the physical phenomena in the cathode and anode areas weakly influence each other and are connected largely by the current through the plasma channel. As the discharge develops between coplanar electrodes, it deposits the positive charge on the dielectric sur- face above the cathode and the negative charge on the surface above the anode. Both processes progress in a wave-like manner with quite pronounced wavefronts.

Let us now compare the properties of the cathode and anode waves.

- Propagation of the cathode wave is, in essence, an expansion of the cathode fall, wherein ionization processes play a determining role. The process of charging the dielectric surface is not a driving force of the propagation of the cathode wave - it can just as easily propagate above the metal. ${ }^{15}$ By contrast, in the anode wave the main process is deposition of the negative charge on the dielectric surface; the deposited charge reverses the vertical component of the electric field near the surface and creates its horizontal component so that the electrons are pulled to still uncharged areas.

- The velocity of the cathode ionizing wave is of the order of the ion velocity in the cathode fall. The velocity of the anode charging wave is independent of the velocity of ions (and almost does not change even when ions are immobile). It is determined by the rate of the delivery of the negative charge (i.e., by the current) through the plasma channel in the area of the anode.

- When the effective thickness $d / \varepsilon$ of the sustain dielectric decreases, the cathode ionizing wave moves faster, and the anode charging wave moves slower (so that it does not propagates at all above the bare electrode).

In the last decade, computers have become so powerful that nowadays it is quite feasible to reach the ultimate goal in simulating PDPs, to track each physical particle (and each photon $)^{14}$ in a PDP cell. This type of code will enable a researcher to focus on optimizing the PDP parameters and to leave the justification of physical approximations behind.

\section{Acknowledgments}

The authors would like to thank L. F. Weber for his numerous comments and suggestions.

\section{Appendix: Time Step in the Numerical Algorithm}

A simple way to get a limitation on the time step $\Delta t$ for recalculation of the electric potential is to consider the equations of the fluid model in which the inertia of electrons is taken into account ${ }^{16}$ :

$$
\begin{gathered}
m n_{e} \frac{d \mathbf{v}}{d t}=-e n_{e} \mathbf{E}-m v_{m}\left(n_{e} \mathbf{v}+D_{e} \nabla n_{e}\right), \\
\frac{\partial n_{e}}{\partial t}=-\operatorname{div}\left(n_{e} \mathbf{v}\right), \\
\operatorname{div} \mathbf{E}=\frac{e}{\varepsilon_{0}}\left(n_{i}-n_{e}\right),
\end{gathered}
$$


where $\mathbf{v}$ is the electron velocity, $n_{e}$ is the electron density, $m$ is the electron mass, $e$ is the electron charge, $v_{m}$ is the momentum transfer collision frequency, and $D_{e}$ is the electron diffusion coefficient. Since slow ions do not impose additional limitations on the time step $\Delta t$, the ion density is assumed constant, $n_{i}=n_{0}$.

Let us consider the evolution of small perturbations of the electron density in the uniform plasma of density $n_{0}$ : $n_{e}=n_{0}+n^{\prime}\left(n^{\prime}<<n_{0}\right)$. From Eqs. (1)-(3), it follows that

$$
\frac{\partial^{2} n^{\prime}}{\partial t^{2}}+v_{m} \frac{\partial n^{\prime}}{\partial t}-v_{m} D_{e} \nabla^{2} n^{\prime}=-\omega_{p e}^{2} n^{\prime}
$$

where $\omega_{p e}=\left(e^{2} n_{0} / \varepsilon_{0} m\right)^{1 / 2}$ is the electron plasma frequency. Leaving the dispersion effects for a more rigorous analysis, we omitted the diffusion term (proportional to $\nabla^{2} n^{\prime}$ ) in Eq. (4) and found the following expression for the frequency $\omega$ of the natural oscillations $[\sim \exp (-i \omega t)]$ :

$$
\omega_{1,2}=-\frac{i}{2} v_{m} \mp \sqrt{\omega_{p e}^{2}-\frac{1}{4} v_{m}^{2}} .
$$

In the case of a strongly collisional plasma $\left(v_{m}>>\omega_{p e}\right)$, we obtain $\omega_{1} \approx-i v_{m}$ and $\omega_{2} \approx-i \omega_{p e}{ }^{2} / v_{m}$. The first eigenmode is related to the relaxation of the electron momentum due to collisions, and it is reproduced in our numerical algorithm by advancing the electrons with a time step $\left(\sim 10^{-14}\right.$ sec) much less than $v_{m}{ }^{-1}$ (which is about $10^{-12}$ sec for a gas mixture used in simulations). The second mode is reproduced in our numerical algorithm by recalculating the Poisson's equation with a time step $\Delta t<\tau_{m}$, where $\tau_{m} \equiv v_{m} / \omega_{p e}{ }^{2}$ is the electron Maxwellian time. Note that in the case of strongly collisional plasma, the time step $\Delta t$ can be taken much greater than $\omega_{p e}{ }^{-1}$.

In the opposite case of weakly collisional plasma $\left(v_{m}<<\right.$ $\left.\omega_{p e}\right)$, the frequencies of the natural oscillations $\omega_{1,2} \approx \mp \omega_{p e}$. In order to suppress the artificial numerical heating of electrons for an extended period of time, one must take the time step $\Delta t$ at least less than $0.2 \omega_{p e}{ }^{-1}$ (the smaller the numerical coefficient the less the artificial heating ${ }^{17}$ ). It useful to note that $\omega_{p e}$ becomes comparable with $v_{m} \sim 10^{12}$ sec at quite a high density of the plasma $n_{0} \sim 3 \times 10^{14} \mathrm{~cm}^{-3}$.

While in the general case one can use expression (A5) to obtain a limitation on the time step, for the sake of sim- plicity we incorporated into the numerical algorithm the following formula for the time step: $\Delta t=\left(\omega_{p e} e^{-1}+\tau_{m}\right) / K$, where a numerical coefficient $K>>1$ and $\omega_{p e} e^{-1}$ and $\tau_{m}$ were evaluated at a point in the volume with a maximum plasma density.

\section{References}

1 J Meunier, Ph Belenguer, and J P Boeuf, "Numerical model of an ac plasma display panel cell in neon-xenon mixtures," J Appl Phys $\mathbf{7 8}$, 731-745 (1995).

2 P J Drallos, V N Khudik, and V P Nagorny, "3D simulations of a single-substrate AC-PDP cell with barrier ribs," SID Symposium Digest 29, 632-635 (1998).

3 G J M Hagelaar, M H Klein, R J M M Snijkers, et al, "Energy loss mechanisms in the microdischarges in plasma display panels," J Appl Phys 89(4), 2033-2039 (2001).

4 Y Hirano, Y Murakami, Y Takano, et al, "3-D computer simulation of spatio-temporal evolution of discharge from writing to sustaining stage in an ac-type pdp cell," Proc IDW '01, 889-892 (2001).

5 V P Nagorny, P J Drallos, and W Williamson, Jr, "The dynamics of a high-pressure ac gas discharge between dielectric coated electrodes near breakdown threshold," J Appl Phys 77, 3645-3656 (1995).

6 V N Khudik, V P Nagorny, and A Shvydky, "Dynamics of a barrier discharge at high overvoltage," J Appl Phys 94, 6291-6302 (2003).

7 D Hayashi, G Heusler, G Hagelaar, et al, "Discharge efficiency in high-Xe-content plasma display panels," J Appl Phys 95, 1656-1661 (2004).

$8 \mathrm{C} \mathrm{H}$ Shon and J K Lee, "Striation phenomenon in the plasma-display panel," Phys Plasmas 8, 1070-1080 (March 2001).

9 A Seguin, L Tessier, H Doyeux, et al, "Measurement of Addressing Speed in Plasma Display Devices,” Proc IDW ‘99, 699-702 (1999).

10 R Ganter, T Callegari, N Posseme, et al, "Photoemission in Plasma Display Panel Discharge Cells," Proc IDW “00, 731-734 (2000).

11 L F Weber, "Status and trends of plasma display device research," presented at EuroDisplay "99, The 19th IDRC, Berlin, Germany (1999). Available at: http://www.plasmadynamics.com

12 J-P Boeuf, "Plasma display panels: physics, recent development and key issues," J Phys D: Appl Phys 36, R53-R79 (2003).

13 G Cho, E-H Choi, Y Seo, et al, "Discharge striations in a coplanar AC-PDP,” J Soc Inf Display 8/3, 211-215 (2000).

14 A Shvydky, V N Khudik, V P Nagorny, et al, "Dynamics of the breakdown in a discharge gap at high overvoltages," $57^{\text {th }}$ Gaseous Electronic Conference, Bunratty, Ireland, Sept. 26-29, 2004.

15 Of course, the dielectric layer can influence the process of the $\mathrm{CF}$ expansion, as can be seen from the fact that in the case of the $\mathrm{CF}$ expansion above the dielectric, the positive-charge deposition occurs primarily in the front of the ionizing wave, whereas in the case of the $\mathrm{CF}$ expansion above the metal, the deposition occurs everywhere below the dense plasma (above the cathode).

16 Yu P Raizer, Gas Discharge Physics (Springer-Verlag, Berlin, New York, 1991)

17 C K Birdsall and A B Langdon, Plasma Physics via Computer Simulation (Bristol, U.K., Adam Hilger, 1991). 